\title{
Role of sleep quality in mediating the relationship between sexual minority status and suicidal behavior among Chinese adolescents
}

This article was published in the following Dove Press journal:

Psychology Research and Behavior Management

\author{
Yeen Huangl,* \\ Pengsheng $\mathrm{Li}^{1}{ }^{1, *}$ \\ Zhisheng Lai \\ Xiaofei Jia ${ }^{3}$ \\ Di Xiao' \\ Tian Wang' \\ Lan Guo ${ }^{2, *}$ \\ Ciyong $\mathrm{Lu}^{1,4, *}$
}

'Department of Medical Statistics and Epidemiology, School of Public Health, Sun Yat-sen University, Guangzhou, Guangdong, People's Republic of China; ${ }^{2}$ Department of HIV/AIDS Prevention and Control, Yuexiu District Center for Disease Control and Prevention, Guangzhou, Guangdong, People's Republic of China; ${ }^{3}$ Department of HIV/ AIDS Prevention and Control, Tianhe District Center for Disease Control and Prevention, Guangzhou, Guangdong, People's Republic of China; ${ }^{4}$ Guangdong Provincial Key Laboratory of Food, Guangdong Engineering Technology Research Center of Nutrition Translation, Sun Yat-sen University, Guangzhou, Guangdong, People's Republic of China

*These authors contributed equally to this work

Correspondence: Ciyong Lu Department of Medical Statistics and Epidemiology, School of Public Health, Sun Yat-sen University, 74 Zhongshan Road 2, Guangzhou, Guangdong 510080 , People's Republic of China

Tel +02087332477

Email luciyong@mail.sysu.edu.cn
Background: Studies suggest that sexual minority adolescents experience higher rates of sleep disturbance than their heterosexual peers, and disturbed sleep is a well-known risk factor for suicidality. This study aimed to explore whether sleep quality had mediating effects on the relationship between sexual minority status and suicidal behavior in Chinese adolescents.

Methods: We analyzed data collected from 7 th to 12 th graders from seven randomly selected provinces of People's Republic of China in the 2015 School-Based Chinese Adolescents Health Survey. The 123,459 students who completed questionnaires regarding sexual attraction, the Pittsburgh Sleep Quality Index, past-year suicidal ideation, and past-year suicide attempts were included in our study (response rate: $95.9 \%$ ).

Results: After adjustment for covariates, sexual minority status was associated with suicidal ideation (adjusted odds ratio $=1.82,95 \% \mathrm{CI}=1.69-1.95$ ) and suicide attempts (adjusted odds ratio $=2.16,95 \% \mathrm{CI}=1.82-2.56$ ). Sleep quality partially mediated the effects of sexual minority status on suicidal ideation (standardized $\beta$ estimate $=0.009,95 \% \mathrm{CI}=0.007-0.012$ ) and suicide attempts (standardized $\beta$ estimate $=0.004,95 \% \mathrm{CI}=0.003-0.005$ ).

Conclusion: Poor sleep quality partially explained the increased risk of suicidality for Chinese sexual minority adolescents. To prevent subsequent suicidality, suicide interventions targeting sexual minorities should be made, with a focus on their disparities in sleep disturbance and sleep health promotion.

Keywords: sexual minority, suicidal behavior, adolescents, sleep quality, mediating effect

\section{Introduction}

Suicidal behavior is the leading cause of injury and death among adolescents worldwide, ${ }^{1}$ which includes suicidal ideation, suicide attempts, and completed suicide. ${ }^{2}$ Although the overall rate of suicide has significantly decreased, People's Republic of China is still a country with high suicide rate, and suicidality remains a threat to the health of Chinese adolescents. ${ }^{3,4}$ An understanding of the risk factors and causes of suicidality is imperative to prevent adolescent suicide.

Many studies showed that sexual minority status (eg, individuals who experience same-sex attraction [SSA]; self-identify as gay, lesbian, or bisexual; or engage in same-sex sexual behavior) is a well-known risk factor for suicidality in adolescents. ${ }^{5,6}$ Meyer's minority stress theory suggests that difficulties in dealing with sexual minority stressors (prejudice, discrimination, and stigma due to same-sex orientation) may lead to a higher risk of suicidality in sexual minority adolescents. ${ }^{7}$ In addition, a previous systematic review showed that a clear majority of studies reported an elevated risk of suicidal ideation and suicide attempts among sexual minority adolescents, ${ }^{8}$ and similar 
results could also be found in Chinese sexual minorities..$^{9,10}$ Thus, there was evidence for a strong relationship between sexual minority status and suicidal behavior in adolescents.

The causes of the elevated risk of suicidality in sexual minority adolescents have not been clearly elucidated. Previous studies have revealed some potentially influence factors (eg, school victimization ${ }^{11}$ and social environment ${ }^{12}$ ) that might mediate the association between sexual minority status and suicidal behavior in adolescents. Sleep plays an important role in the growth of adolescents, and poor sleep quality may increase the risk of physical and psychological health consequences for them. ${ }^{13}$ A National Health Interview Survey from the United States showed that sexual minorities reported poorer sleep quality than their heterosexual peers. ${ }^{14}$ Moreover, cross-sectional and longitudinal studies have also illustrated that sleep quality is significantly associated with suicidality. ${ }^{15-17}$ Therefore, it is reasonable to hypothesize that sleep quality may play a mediating role in the association between sexual minority status and suicidal behavior; however, no previous study has examined that mediating role.

Thus, we analyzed data collected from the 2015 SchoolBased Chinese Adolescents Health Survey (SCAHS) to test the mediating effects of sleep quality on the relationship between sexual minority status and suicidal behavior in Chinese adolescents. We hypothesized that sexual minority status was significantly associated with suicidal behavior, and that this relationship was partially mediated by sleep quality.

\section{Methods}

\section{Study sample and data collection}

In this study, we employed data from the 2015 SCAHS. ${ }^{18,19}$ It is an ongoing, large-scale, health-related behavior survey conducted among Chinese adolescents (grades 7-12) biennially since 2007 . The 2015 survey was the latest one conducted in seven provinces in People's Republic of China. ${ }^{20}$ Sampling procedure of the 2015 SCAHS has been described in our previous studies. ${ }^{21,22}$ In total, 150,822 students finished the questionnaires (the response rate was 95.9\%). Among all the students, 27,363 students responded "unsure" (17.3\%) to the sexual minority status question, which was greater than in previous studies; those "unsure" students probably included not only individuals who were still unsure of their sexual orientation but also heterosexual students who were unwilling to disclose their sexual orientation or did not understand the question because of their younger age, which might result in an underestimation of the suicidality disparities in our study. Therefore, students belonging to the "unsure" category were not involved in the analysis and we mainly focused on the suicidality disparities of students who had already acknowledged their SSA. Therefore, a total of 123,459 students were included in this study.

A standardized self-administered questionnaire was given to each student from the selected classes on the survey day to be finished in the classroom within a class period (normally 40 or 45 minutes). For the sake of the students' privacy, all questionnaires were completed anonymously by students without the presence of any teachers or school personnel (in order to avoid potential information bias).

\section{Ethical statement}

This study was conducted in accordance with the Declaration of Helsinki and was approved by the Institutional Review Board of the School of Public Health of Sun Yat-sen University. Written informed consent was acquired from every participating student who had already reached the age of 18 years and from one parent (or legal guardian) of each participating student under the age of 18 years.

\section{Measures \\ Sexual minority status}

Sexual minority status was measured by asking students the following question regarding sexual attraction: "In a romantic relationship, which kind of person are you attracted to?" Response options included the following: "opposite-sex", "same-sex", "equally opposite-sex and same-sex", and "unsure". 5,23,24 Students who reported "same-sex" or "equally opposite-sex and same-sex" attraction were classified as SSA and both-sex attraction (BSA). Students who belonged to SSA and BSA were classified as sexual minorities. The same question has been used in previous studies among Chinese adolescents. ${ }^{21,24}$

\section{Suicidal behavior}

Suicidal behavior included suicidal ideation and suicide attempts. Suicidal ideation was assessed by the question: "During the past 12 months, how many times did you seriously consider attempting suicide?" Suicide attempts were measured by asking the question: "During the past 12 months, how many times did you actually attempt suicide?" Students were asked to rate these two questions on a scale of zero or once or at least twice. ${ }^{25,26}$

\section{Sleep quality}

The Chinese version of the Pittsburgh Sleep Quality Index (PSQI) was used to assess the students' sleep quality over the previous month. ${ }^{27}$ The PSQI contains seven components (sub- 
jective sleep quality, sleep latency, sleep duration, habitual sleep efficiency, sleep disturbance, use of sleep medications, and daytime dysfunction), and the score for each component ranges from 0 to 3 points. The global PSQI score ranges from 0 to 21 , with higher scores indicating poorer sleep quality. ${ }^{21}$ The PSQI has been demonstrated to be valid and reliable in the Chinese population. ${ }^{28}$

\section{Covariates}

Covariates that have been previously reported to be associated with suicidal behavior among sexual minority adolescents were taken into account. ${ }^{5,29-31}$ These covariates included age, biological sex, living arrangement, household socioeconomic status (HSS), academic pressure, classmate relationship, teacher-classmate relationships, smoking, alcohol consumption, school victimization, and depressive symptoms.

The living arrangement was measured by asking the student who lived in his or her primary home ("living with both parents", "living with father or mother only", and "living with others"). HSS was estimated by asking about the students' perceptions of their own household's current socioeconomic status ("good", "average", and "poor"). Academic pressure was measured based on the students' self-rating of their own school work ("none", "less", and "medium or great"). Classmate relationships and teacher-classmate relationships were evaluated according to the students' self-rated relationships with their classmates and teachers ("good", "average", and "poor"). Smoking was defined as smoking $\geq 1$ cigarettes in the past 30 days. ${ }^{32}$ Alcohol consumption was defined as drinking alcohol for $\geq 1$ day in the past 30 days. ${ }^{33}$ School victimization was assessed based on the definition of bullying in the Olweus Bully/Victim Questionnaire. ${ }^{34}$ Students were asked to answer the following question: "How frequently have you been bullied at school in the previous month?" The answers were "none", "1-2 days", "3-5 days", "6-9 days", "10-19 days", and "more than 20 days". Students who reported being bullied for three or more days in the previous month were regarded as experiencing school victimization. ${ }^{21,35}$ Depressive symptoms were assessed with the Chinese version of the Depression Self-Rating Scale for Children (DSRSC) to determine whether students had any depressive symptoms..$^{36}$ The score range of the DSRSC was $0-54$ points, and scores that were $>15$ points suggested the presence of depressive symptoms. This scale had been proven to be both valid and reliable in Chinese adolescents. ${ }^{37}$

\section{Statistical analyses}

In this study, prevalence estimates and association analyses accounted for the complex sampling design, which included sampling strata, clusters, and weights. The sampling weights were calculated according to student biological sex, type of school, and student grade, and the weight information was acquired from the Educational Statistics Yearbook of China 2013. First, descriptive analyses were conducted to describe the demographic characteristics, sleep quality, suicidal ideation, and suicide attempts in both heterosexual and sexual minority adolescents; the Rao-Scott $\chi^{2}$ and $t$-tests were utilized to compare the differences between groups. Second, univariate logistic regression models were generated to examine the relationship between sexual minority status and suicidal ideation and suicide attempts. The variables that were significant at the 0.10 level in univariate analyses or were widely reported in previous studies were simultaneously entered in the multivariate logistic regression models. OR, adjusted odds ratios (AORs), and $95 \% \mathrm{CI}$ were obtained from the logistic regression models. Third, structural equation modeling (SEM) models using the maximum likelihood (ML) method were utilized to examine the potential mediating role of sleep quality on the association of sexual minority status with suicidal ideation and suicide attempts (Figure 1). Several commonly applied model fit indices were recommended to evaluate the SEM models: 1) the comparative fit index (CFI $>0.90$ indicates good fit), 2) the standardized root mean square residual (SRMR $<0.08$ indicates good fit), and 3 ) the root mean square error of approximation (RMSEA $<0.08$ indicates acceptable fit). ${ }^{38}$ Standardized probit coefficients, standardized indirect effects, and standardized total effects were reported, and the bias-corrected $95 \%$ CI was estimated using 5,000 bootstrap samples. ${ }^{39}$ Missing data in the analysis accounted for $<3.1 \%$ for all relevant variables, and observations with missing data were eliminated. A twotailed $P$-value $<0.05$ was considered statistically significant. All statistical analyses were conducted using SAS 9.3 (SAS Institute Inc., Cary, NC, USA) and Mplus V.7.4 (Muthén and Muthén, Los Angeles, CA, USA).

\section{Results \\ Characteristics of participants stratified by sexual minority status}

The sample characteristics are shown in Table 1. Among the 123,459 students included in the study, the mean (SE) age of the students was $15.3(0.5)$ years; $95.0 \%$ of the included students self-reported as heterosexuals, while $1.6 \%$ and $3.4 \%$ self-reported as SSA and BSA, respectively. Female students were more likely to report SSA than male students $(P<0.001)$. Sexual minority students were more likely to report school victimization $(16.1 \%$ vs $8.0 \%, P<0.001)$ and 


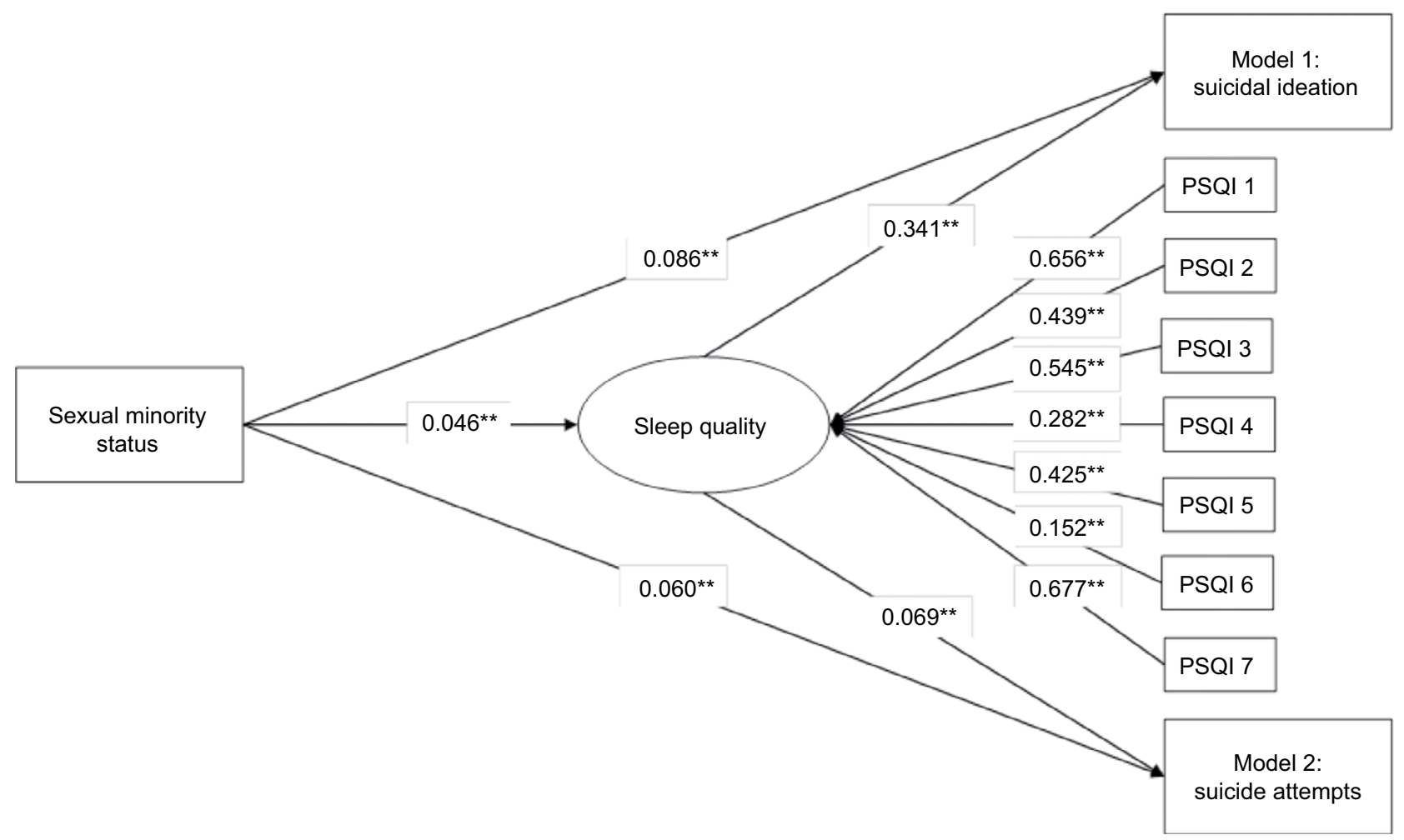

Figure I SEMs for the relationship between sexual minority status and suicidal behavior.

Notes: PSQI I represents subjective sleep quality; PSQI 2 represents sleep latency; PSQI 3 represents sleep duration; PSQI 4 represents habitual sleep efficiency; PSQI 5 represents sleep disturbance; PSQI 6 represents use of sleeping medication; and PSQI 7 represents daytime dysfunction. Model I means the structural equation model for suicidal ideation, and Model 2 represents the model for suicide attempts. Sexual minority status was defined as adolescents who reported same-sex or both-sex attraction. Sleep quality was measured by the global PSQI score, and seven PSQI individual components scores (PSQI I-7) were incorporated to set up a latent variable (global PSQI score) in the SEM. Suicidal ideation was defined as reporting having one or more times seriously considered attempting suicide during the past 12 months. Suicide attempts were defined as reporting having one or more times actually attempted suicide during the past 12 months. ${ }^{* * P}<0.00 \mathrm{I}$.

Abbreviations: PSQI, Pittsburgh Sleep Quality Index; SEM, structural equation model.

depressive symptoms $(32.4 \%$ vs $17.6 \%, P<0.001)$ than their heterosexual peers. Furthermore, sexual minority students had significantly higher global PSQI scores (6.3 vs 5.3, $P<0.001)$ than their heterosexual peers. The weighted prevalence of having past-year suicidal ideation $(35.2 \%$ vs $16.6 \%$, $P<0.001)$ and suicide attempts $(10.2 \%$ vs $2.6 \%, P<0.001)$ were significantly higher in sexual minority students than in heterosexual students.

\section{Associations of sexual minority status with sleep quality and suicidal behavior}

As shown in Table 2, in the univariable logistic regression models, sexual minority status was significantly associated with poor sleep quality and past-year suicidal ideation and suicide attempts $(P<0.001)$. Furthermore, after the adjustment for age, biological sex, living arrangement, HSS, academic pressure, classmate relationships, teacher-classmate relationships, smoking, alcohol consumption, school victimization, and depressive symptoms, our multivariable logistic regression models revealed that sexual minority status was still associated with poor sleep quality (BSA:
$\mathrm{AOR}=1.54,95 \% \mathrm{CI}=1.43-1.64)$ and past-year suicidal ideation (SSA: AOR $=1.31,95 \% \mathrm{CI}=1.14-1.50$; BSA: $\mathrm{AOR}=2.07,95 \% \mathrm{CI}=1.88-2.28)$ and suicide attempts (SSA: AOR=2.04, 95\% CI=1.59-2.62; BSA: $\mathrm{AOR}=2.20$, $95 \% \mathrm{CI}=1.79-2.70)$.

\section{Mediating effects of sleep quality}

The standardized path coefficients and standardized effects for past-year suicidal ideation and suicide attempts are shown in Table 3. Model fit indices of structural equation models indicated good fit for suicidal ideation (Model $1 \mathrm{fit}$ indices: $\mathrm{CFI}=0.935$; RMSEA $=0.047,95 \% \mathrm{CI}=0.046-0.048$; $\mathrm{SRMR}=0.016$ ) and suicide attempts (Model 2 fit indices: $\mathrm{CFI}=0.931$; $\mathrm{RMSEA}=0.046,95 \% \mathrm{CI}=0.046-0.047$; $\mathrm{SRMR}=0.015)$ as dependent variables, respectively. The direct standardized path coefficients of the effects of sexual minority status on sleep quality (standardized $\beta$ estimate $=0.046,95 \% \mathrm{CI}=0.033-0.057$ ) and the effects of sleep quality on suicidal ideation (standardized $\beta$ estimate $=0.252,95 \% \mathrm{CI}=0.244-0.261)$ and on suicide attempts (standardized $\beta$ estimate $=0.112,95 \%$ CI $=0.099-0.124$ ) 
Table I Sample characteristics stratified by sexual minority status $(\mathrm{N}=123,459)$

\begin{tabular}{|c|c|c|c|c|}
\hline \multirow[t]{2}{*}{ Variables } & \multirow{2}{*}{\begin{tabular}{|l|} 
Total \\
$\mathbf{N}(\%)$
\end{tabular}} & \multirow{2}{*}{$\begin{array}{l}\text { Heterosexuals } \\
\mathbf{N}(\%)\end{array}$} & \multirow{2}{*}{$\begin{array}{l}\text { Sexual minorities }^{\mathrm{a}} \\
\mathbf{N}(\%)\end{array}$} & \multirow[t]{2}{*}{$\chi^{2 / t}$} \\
\hline & & & & \\
\hline Total & $123,459(100.0)$ & $116,774(95.0)$ & $6,685(5.0)$ & \\
\hline \multicolumn{5}{|l|}{ Sexual minority status } \\
\hline Heterosexuals & I I 6,774 (95.0) & NA & NA & NA \\
\hline Same-sex attraction & $2,053(1.6)$ & NA & NA & \\
\hline Both-sex attraction & $4,632(3.4)$ & NA & NA & \\
\hline Age, mean (SE) & I5.3(0.5) & $15.3(0.5)$ & I5.I (0.4) & $7.90^{* *}$ \\
\hline Biological sex & & & & $169.35^{* *}$ \\
\hline Male & $59,826(52.2)$ & $57,343(52.8)$ & $2,483(4 I .4)$ & \\
\hline Female & $63,633(47.8)$ & $59,431(47.2)$ & $4,202(58.6)$ & \\
\hline Living arrangement & & & & $|3.3|^{* *}$ \\
\hline Living with parents & $89,210(74.5)$ & $84,54 \mid(74.6)$ & $4,669(72.2)$ & \\
\hline Living with a single parent & $14,800(1 \mid .2)$ & I3,897 (II.I) & $903(12.4)$ & \\
\hline Living with others & $19,449(14.3)$ & $18,336(14.3)$ & $\mathrm{I}, \mathrm{II}(\mathrm{I}(\mathrm{I} .4)$ & \\
\hline HSS & & & & $60.73^{* *}$ \\
\hline Good & $24,268(21.9)$ & $22,812(21.8)$ & $\mathrm{I}, 456(24.2)$ & \\
\hline Average & $75,054(60.9)$ & $71,306(61.1)$ & $3,748(55.4)$ & \\
\hline Poor & $24,137(17.2)$ & $22,656(17.1)$ & $\mathrm{I}, 48 \mathrm{I}(20.4)$ & \\
\hline Academic pressure & & & & $64.22^{* *}$ \\
\hline None & $18, I I I(15.0)$ & $17,05 \mid(14.9)$ & $1,060(16.1)$ & \\
\hline Less & $55,709(45.7)$ & $53,06 \mid(46.1)$ & $2,648(39.9)$ & \\
\hline Medium or great & $49,639(39.3)$ & $46,662(39.0)$ & $2,977(44.0)$ & \\
\hline Classmate relationships & & & & $173.48^{* *}$ \\
\hline Good & $91,293(75.3)$ & $86,695(75.6)$ & $4,598(69.4)$ & \\
\hline Average & $30,234(23.1)$ & $28,452(23.0)$ & $1,782(25.8)$ & \\
\hline Poor & $1,932(1.6)$ & $\mathrm{I}, 627(\mathrm{I} .4)$ & $305(4.8)$ & \\
\hline Teacher-classmate relationships & & & & $124.63^{\text {** }}$ \\
\hline Good & $67,465(55.8)$ & $64,192(56.1)$ & $3,273(49.6)$ & \\
\hline Average & $5 \mathrm{I}, 875(41.0)$ & $48,946(40.8)$ & $2,929(43.0)$ & \\
\hline Poor & $4,119(3.2)$ & $3,636(3.1)$ & $483(7.4)$ & \\
\hline Smoking & & & & $10.89^{* *}$ \\
\hline No & $116,144(93.9)$ & $109,954(93.9)$ & $6,190(92.6)$ & \\
\hline Yes & $7,315(6.1)$ & $6,820(6.1)$ & $495(7.4)$ & \\
\hline Alcohol consumption & & & & $40.68^{* *}$ \\
\hline No & $10 I, 98 \mid(82.6)$ & $96,909(82.9)$ & $5,072(76.6)$ & \\
\hline Yes & $21,478(17.4)$ & $19,865(17.1)$ & $\mathrm{I}, 613(23.4)$ & \\
\hline School victimization & & & & $253.17^{* *}$ \\
\hline No & II $3,435(9 \mid .6)$ & $107,767(92.0)$ & $5,668(83.9)$ & \\
\hline Yes & $10,024(8.4)$ & $9,007(8.0)$ & $1,017(16.1)$ & \\
\hline Depressive symptoms & & & & $677.58^{\text {*k }}$ \\
\hline No & $99,637(81.7)$ & $95,183(82.4)$ & $4,454(67.6)$ & \\
\hline Yes & $23,822(18.3)$ & $21,591(17.6)$ & $2,231(32.4)$ & \\
\hline Sleep quality ${ }^{\mathrm{b}}$, mean (SE) & $5.4(0.3)$ & $5.3(0.3)$ & $6.3(0.3)$ & $26.10^{* *}$ \\
\hline Suicidal ideation $^{c}$ & & & & $1,411.58^{* *}$ \\
\hline No & $100,772(82.5)$ & $96,513(83.4)$ & $4,259(64.8)$ & \\
\hline Yes & $22,687(17.5)$ & $20,261(16.6)$ & $2,426(35.2)$ & \\
\hline Suicide attempts $^{d}$ & & & & $1,183.42^{* *}$ \\
\hline No & $119,515(97.0)$ & I I 3,543 (97.4) & $5,972(89.8)$ & \\
\hline Yes & $3,944(3.0)$ & $3,231(2.6)$ & $713(10.2)$ & \\
\hline
\end{tabular}

Notes: All numbers were unweighted, where all percentages were adjusted for sampling weights. ${ }^{2}$ Sexual minorities included adolescents who reported same-sex or both-

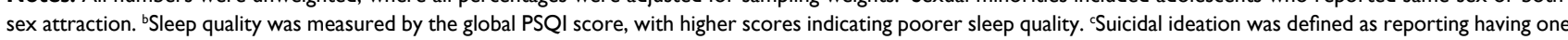
or more times seriously considered attempting suicide during the past 12 months. SSuicide attempts were defined as reporting having one or more times actually attempted suicide during the past 12 months. " $P<0.001$.

Abbreviations: HSS, household socioeconomic status; No., number; NA, not applicable or no data available; PSQI, Pittsburgh Sleep Quality Index. 
Table 2 Associations of sexual minority status with sleep quality and suicidal behavior among Chinese adolescents $(\mathrm{N}=123,459)$

\begin{tabular}{|c|c|c|c|c|c|c|}
\hline \multirow[t]{2}{*}{ Variables } & \multicolumn{2}{|c|}{ Poor sleep quality } & \multicolumn{2}{|l|}{ Suicidal ideation ${ }^{\mathrm{b}}$} & \multicolumn{2}{|c|}{ Suicide attempts ${ }^{c}$} \\
\hline & $\begin{array}{l}\text { Model I: } \\
\text { OR }(95 \% \mathrm{Cl})\end{array}$ & $\begin{array}{l}\text { Model 2: } \\
\text { AOR }(95 \% \mathrm{Cl})\end{array}$ & $\begin{array}{l}\text { Model I: } \\
\text { OR }(95 \% \mathrm{Cl})\end{array}$ & $\begin{array}{l}\text { Model 2: } \\
\text { AOR }(95 \% \mathrm{Cl})\end{array}$ & $\begin{array}{l}\text { Model I: } \\
\text { OR }(95 \% \mathrm{Cl})\end{array}$ & $\begin{array}{l}\text { Model 2: } \\
\text { AOR }(95 \% \mathrm{Cl})\end{array}$ \\
\hline \multicolumn{7}{|l|}{$\begin{array}{l}\text { Sexual } \\
\text { minority status }\end{array}$} \\
\hline Heterosexuals & 1.00 & 1.00 & 1.00 & 1.00 & 1.00 & 1.00 \\
\hline $\begin{array}{l}\text { Same-sex } \\
\text { attraction }\end{array}$ & $1.24(1.12-1.36)^{* * *}$ & $1.05(0.94-1.17)$ & $1.73(1.50-2.00)^{* *}$ & $1.31(1.14-1.50)^{* *}$ & $3.17(2.53-3.98)^{* *}$ & $2.04(1.59-2.62)^{* *}$ \\
\hline $\begin{array}{l}\text { Both-sex } \\
\text { attraction }\end{array}$ & $2.00(1.88-2.13)^{* * k}$ & $1.54(1.43-1.64)^{* *}$ & $3.31(2.98-3.67)^{* *}$ & $2.07(1.88-2.28)^{* *}$ & $4.82(3.85-6.04)^{* *}$ & $2.20(1.79-2.70)^{* k}$ \\
\hline
\end{tabular}

Notes: Model I was unadjusted. Model 2 was adjusted for age, biological sex, living arrangement, household socioeconomic status, academic pressure, classmate relationships,

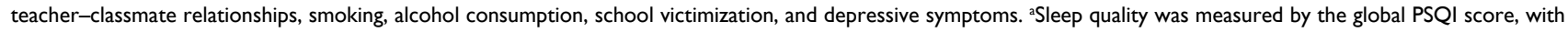

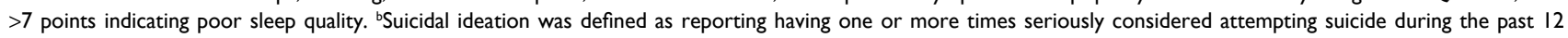
months. 'Suicide attempts were defined as reporting having one or more times actually attempted suicide during the past 12 months. ${ }^{* * P}<0.00 \mathrm{I}$.

Abbreviations: AOR, adjusted odds ratio; PSQI, Pittsburgh Sleep Quality Index.

Table 3 Mediating effects of sleep quality on the relationship between sexual minority status and suicidal behavior among Chinese adolescents $(\mathrm{N}=123,459)$

\begin{tabular}{|l|l|l|}
\hline Variables & $\begin{array}{l}\text { Model I: } \\
\text { suicidal ideation }^{\mathrm{a}}\end{array}$ & $\begin{array}{l}\text { Model 2: } \\
\text { suicide }^{\text {attempts }^{\mathrm{b}}}\end{array}$ \\
\hline Path & & \\
\hline Sexual minority status \\
\hline Sleep quality $\rightarrow$ Suicidal behavior & $0.046(0.033-0.057)^{* *}$ & $0.046(0.033-0.057)^{* *}$ \\
\hline Sexual minority status $\rightarrow$ Suicidal behavior & $0.252(0.244-0.26)^{* * *}$ & $0.112(0.099-0.124)^{* *}$ \\
\hline Standardized effect & $0.086(0.073-0.099)^{* * *}$ & $0.060(0.048-0.074)^{* *}$ \\
\hline Indirect effect & & \\
\hline Total effect & $0.009(0.007-0.012)^{* * *}$ & $0.004(0.003-0.005)^{* * *}$ \\
\hline
\end{tabular}

Notes: The structural equation models for suicidal ideation (model I) and suicide attempts (model 2) were adjusted for age, biological sex, living arrangement, household socioeconomic status, academic pressure, classmate relationships, teacher-classmate relationships, smoking, alcohol consumption, school victimization, and depressive symptoms. Model I fit indices: $\mathrm{CFI}=0.935$; RMSEA=0.047, 95\% Cl=0.046-0.048; SRMR=0.016; Model 2 fit indices: $\mathrm{CFI}=0.93 \mathrm{I}$; $\mathrm{RMSEA}=0.046,95 \% \mathrm{Cl}=0.046-0.047$; $S R M R=0.015$. aSuicidal ideation was defined as reporting having one or more times seriously considered attempting suicide during the past 12 months. ${ }^{b}$ Suicide attempts were defined as reporting having one or more times actually attempted suicide during the past 12 months. 'Sexual minority status was defined as adolescents who reported same-sex or both-sex attraction. `Sleep quality was measured by the global PSQI score, with higher scores indicating poorer sleep quality. $* * P<0.00 \mathrm{I}$.

Abbreviations: CFI, comparative fit index; PSQI, Pittsburgh Sleep Quality Index; RMSEA, root mean square error of approximation; SRMR, standardized root mean square residual.

were statistically significant $(P<0.001)$. The standardized indirect effects of sexual minority status on suicidal ideation ( standardized $\beta$ estimate $=0.009,95 \% \mathrm{CI}=0.007-0.012$ ) and on suicide attempts (standardized $\beta$ estimate $=0.004,95 \%$ $\mathrm{CI}=0.003-0.005)$ mediated by sleep quality were statistically significant $(P<0.001)$.

\section{Discussion}

Our study found that poor sleep quality partially explained the elevated risk of suicidality in Chinese sexual minority adolescents. To our knowledge, this is the first national, largescale study to examine the potential mediating role of sleep quality on the relationship between sexual minority status and suicidal behavior. Our findings provide a new hypothesis for understanding the process of suicidality in sexual minorities, which might help in the early detection of and targeted intervention in suicidality among those high-risk individuals.
We found that sexual minority adolescents experienced a higher prevalence of past-year suicidal ideation and suicide attempts than their heterosexual peers. Moreover, after adjustment for demographic characteristics, school victimization, and depressive symptoms, sexual minority status was still associated with suicidal behavior in adolescents. Our findings supported the findings of previous studies on the ubiquity of an elevated suicidality risk among sexual minorities ${ }^{5,29}$ and suggested that Chinese sexual minority adolescents are more vulnerable to suicidal ideation and suicide attempts than their heterosexual peers.

Our results revealed that sexual minority adolescents reported poorer sleep quality than their heterosexual peers. After adjustment for covariates, poor sleep quality was still associated with suicidal behavior. These results are consistent with those of previous studies. ${ }^{14,40}$ More importantly, Meyer's minority stress model illustrated how stigma related to sexual 
minority status affected sexual minorities' mental health through mediators such as distal/proximal stressors (ie, status $\rightarrow$ stress $\rightarrow$ psychopathology). ${ }^{7}$ Based on these minority stress theories, our study provided a novel hypothesis that was tested in our national, large-scale sample; the results showed that sleep quality played a potentially mediating role in the association between sexual minority status and suicidal behavior. To the best of our knowledge, no previous studies have explored these mediating effects. One potential explanation for these associations is that poor sleep is a warning sign of suicidality. ${ }^{41}$ Two previous reviews indicated that sleep disturbances such as insomnia, nightmares, and poor sleep quality symptoms may result in an elevated risk of suicidal ideation and suicide attempts. ${ }^{42,43}$ Moreover, the minority stress model suggests that sexual minorities are at increased risk of mental health burdens due to difficulties in dealing with minority stressors (eg, prejudice, discrimination, and violence) ${ }^{7}$ these stigmarelated stressors create elevations in emotion dysregulation and social/interpersonal problems (eg, social isolation and social norms), and these processes, in turn, mediate the relationship between stigma-related stressors and mental health in sexual minorities (ie, status $\rightarrow$ stress $\rightarrow$ psychological mediators $\rightarrow$ psychopathology). ${ }^{44}$ A previous study showed that social isolation and psychological distress can lead to disturbed sleep, ${ }^{45}$ and sexual minorities may also develop poor sleep quality from these adverse experiences. Therefore, sexual minorities may experience sleep disturbances caused by their sexual minority stressors, and poorer sleep quality could lead to an increased risk of suicidality.

Although the underlying causes of the association between sexual minority status and suicidal behavior are complex and remain unclear, our study provided a novel possible explanation for the mediating effects of sleep quality on this association. Our findings suggest the following interventions to prevent suicidality in sexual minority adolescents: 1) government and public health organizations should establish a nationwide active surveillance system (such as the Youth Risk Behavior Surveillance System in the United States) to monitor suicidality in Chinese sexual minority adolescents; 2) schools and related education departments should create a positive school climate through formulating "safe school" policies and programs (eg, anti-discrimination and antiharassment policies) to reduce prejudice, discrimination, and school violence toward sexual minorities; ${ }^{46} 3$ ) parents and guardians should help their sexual minority children in promoting acceptance of their sexual orientation and pay particular attention to promoting sleep health education (eg, developing good sleep habits) for their children; ${ }^{47} 4$ ) suicide-related interventions targeting sexual minorities should investigate methods (eg, measurement of levels of serotonin) of early detection of those who have disturbed sleep (eg, serotonergic dysregulation, irregular sleep, and social rhythms); ${ }^{42}$ and 5) psychologists, psychiatrists, and related clinical workers should focus on sexual minorities who have sleep disturbances (eg, insomnia, nightmares, and poor sleep quality symptoms) and develop appropriate treatments (eg, pharmacological and cognitive-behavioral treatment $)^{48}$ targeting poor sleep, so as to help the early detection of suicidal behavior and to prevent subsequent suicidality in those high-risk individuals.

Several limitations of this study should be noted when interpreting the results. First, the cross-sectional design of this study limited our ability to make causal inferences. Second, in our study, students' sleep quality was measured by the PSQI scale without corroborating clinical measurement, which could not completely rule out the possibility of recall bias. Third, our study sample included students attending school but did not include adolescents who had dropped out of school or were absent from school on the day the survey was administered; poor sleep quality and suicidal behavior may be more common among the students who were absent. Fourth, although our logistic regression model and SEM models were corrected for important covariates (eg, school victimization and depressive symptoms), other potential confounding factors (eg, genetics, anxiety, and bipolar disorder) that may influence sleep quality and suicidality were not included in our study.

\section{Conclusion}

The findings from this study indicated that Chinese sexual minority adolescents have poorer sleep quality than their heterosexual peers, and poor sleep quality partially explained the increased risk of suicidality in sexual minority adolescents. Suicide interventions targeting sexual minorities should be made, with close attention to their sleep disturbances, and appropriate treatment strategies for those experiencing poor sleep quality to promote their sleep health. These may help in the early detection and prevention of suicidality in sexual minorities.

\section{Acknowledgments}

We thank the local health professionals, Department of Education, and participating schools for their assistance and support. In addition, we also express our gratitude to the participating investigators (Ming Li, Siyuan Pan, and Wuquan Li) for assisting in collecting data and Qiaohong Chen for providing language help. This work was supported by the National Natural Science Foundation of China (grant number 81673252) 
and the Natural Science Foundation of Guangdong Province, People's Republic of China (grant number 2014A030313174).

\section{Author contributions}

CL conceptualized and designed the study, reviewed as well as revised the manuscript. YH and PL conceptualized and designed the study, coordinated and supervised the data collection, carried out the initial analyses, and drafted the initial manuscript. They contributed equally to this study. LG carried out the analyses and interpreted the data, reviewed and revised the manuscript. ZL, XJ, DX, and TW designed the data collecting instruments, coordinated the supervision of data collecting, and reviewed and revised the manuscript. All authors contributed to data analysis, drafting and revising the article, gave final approval of the version to be published, and agree to be accountable for all aspects of the work.

\section{Disclosure}

The authors report no conflicts of interest in this work.

\section{References}

1. World Health Organization. Suicide Rates per 100,000 by Country, Year and Sex (Table). Geneva 27. Switzerland: World Health Organization; 2015. Available from: http://www.who.int/mental_health/prevention/ suicide/suicideprevent/en/. Accessed November 30, 2018.

2. Nock MK, Borges G, Bromet EJ, Cha CB, Kessler RC, Lee S. Suicide and suicidal behavior. Epidemiol Rev. 2008;30:133-154.

3. Zhang J, Sun L, Liu Y, Zhang J. The change in suicide rates between 2002 and 2011 in China. Suicide Life Threat Behav. 2014;44(5): $560-568$.

4. Cui S, Cheng Y, Xu Z, Chen D, Wang Y. Peer relationships and suicide ideation and attempts among Chinese adolescents. Child Care Health Dev. 2011;37(5):692-702.

5. Russell ST, Joyner K. Adolescent sexual orientation and suicide risk: evidence from a national study. Am J Public Health. 2001;91(8):1276-1281.

6. Wichstrøm L, Hegna K. Sexual orientation and suicide attempt: a longitudinal study of the general Norwegian adolescent population. $J$ Abnorm Psychol. 2003;112(1):144-151.

7. Meyer IH. Prejudice, social stress, and mental health in lesbian, gay, and bisexual populations: conceptual issues and research evidence. Psychol Bull. 2003;129(5):674-697.

8. Plöderl M, Tremblay P. Mental health of sexual minorities. A systematic review. Int Rev Psychiatry. 2015;27(5):367-385.

9. $\mathrm{Mu} \mathrm{H}, \mathrm{LiY}$, Liu L, et al. Prevalence and risk factors for lifetime suicide ideation, plan and attempt in Chinese men who have sex with men. $B M C$ Psychiatry. 2016;16:117.

10. Chen H, Li Y, Wang L, Zhang B. Causes of suicidal behaviors in men who have sex with men in China: a national questionnaire survey. $B M C$ Public Health. 2015;15:91.

11. Burton CM, Marshal MP, Chisolm DJ, Sucato GS, Friedman MS. Sexual minority-related victimization as a mediator of mental health disparities in sexual minority youth: a longitudinal analysis. $J$ Youth Adolesc. 2013;42(3):394-402.

12. Hatzenbuehler ML. The social environment and suicide attempts in lesbian, gay, and bisexual youth. Pediatrics. 2011;127(5):896-903.

13. Carskadon MA, Acebo C, Jenni OG. Regulation of adolescent sleep: implications for behavior. Ann N Y Acad Sci. 2004;1021:276-291.
14. Galinsky AM, Ward BW, Joestl SS, Dahlhamer JM. Sleep duration, sleep quality, and sexual orientation: findings from the 2013-2015 National Health Interview Survey. Sleep Health. 2018;4(1):56-62.

15. Bernert RA, Turvey CL, Conwell Y, Joiner TE. Association of poor subjective sleep quality with risk for death by suicide during a 10 -year period: a longitudinal, population-based study of late life. JAMA Psychiatry. 2014;71(10):1129-1137.

16. Qian Y, Sun L, Zhou C, Ge D, Zhang L. The association between suicidal ideation and sleep quality in elderly individuals: a cross-sectional study in Shandong, China. Psychiatry Res. 2017;256:453-457.

17. Gelaye B, Okeiga J, Ayantoye I, Berhane HY, Berhane Y, Williams MA. Association of suicidal ideation with poor sleep quality among Ethiopian adults. Sleep Breath. 2016;20(4):1319-1326.

18. Wang H, Deng J, Zhou X, et al. The nonmedical use of prescription medicines among high school students: a cross-sectional study in Southern China. Drug Alcohol Depend. 2014;141:9-15.

19. Guo L, Xu Y, Deng J, et al. Non-medical use of prescription pain relievers among high school students in China: a multilevel analysis. $B M J$ Open. 2015;5(7):e007569.

20. Guo L, Wang W, Gao X, Huang G, Li P, Lu C. Associations of childhood maltreatment with single and multiple suicide attempts among older Chinese adolescents. J Pediatr. 2018;196:244-250.e1.

21. Li P, Huang Y, Guo L, et al. Is sexual minority status associated with poor sleep quality among adolescents? Analysis of a national cross-sectional survey in Chinese adolescents. BMJ Open. 2017;7(12):e017067.

22. Huang Y, Li P, Lai Z, et al. Association between sexual minority status and suicidal behavior among Chinese adolescents: a moderated mediation model. J Affect Disord. 2018;239:85-92.

23. Saewyc EM, Bauer GR, Skay CL, et al. Measuring sexual orientation in adolescent health surveys: evaluation of eight school-based surveys. J Adolesc Health. 2004;35(4):345.e1-15.

24. Li P, Huang Y, Guo L, et al. Sexual attraction and the nonmedical use of opioids and sedative drugs among Chinese adolescents. Drug Alcohol Depend. 2018;183:169-175.

25. Guo L, Xu Y, Deng J, et al. Association between nonmedical use of prescription drugs and suicidal behavior among adolescents. JAMA Pediatr. 2016;170(10):971-978.

26. Woods ER, Lin YG, Middleman A, Beckford P, Chase L, Durant RH. The associations of suicide attempts in adolescents. Pediatrics. 1997;99(6):791-796.

27. Buysse DJ, Reynolds CF 3rd, Monk TH, Berman SR, Kupfer DJ. The Pittsburgh Sleep Quality Index: a new instrument for psychiatric practice and research. Psychiatry Res. 1989;28(2):193-213.

28. Liu XC, Tang MQ, Hu L, et al. Reliability and validity of the Pittsburgh Sleep Quality Index. Chin J Psychiatry. 1996;29(2):103-107. Chinese.

29. Remafedi G, French S, Story M, Resnick MD, Blum R. The relationship between suicide risk and sexual orientation: results of a populationbased study. Am J Public Health. 1998;88(1):57-60.

30. Shields JP, Whitaker K, Glassman J, Franks HM, Howard K. Impact of victimization on risk of suicide among lesbian, gay, and bisexual high school students in San Francisco. J Adolesc Health. 2012;50(4):418-420.

31. Denny S, Lucassen MF, Stuart J, et al. The association between supportive high school environments and depressive symptoms and suicidality among sexual minority students. J Clin Child Adolesc Psychol. 2016;45(3):248-261.

32. Acierno R, Kilpatrick DG, Resnick H, Saunders B, De Arellano M, Best C. Assault, PTSD, family substance use, and depression as risk factors for cigarette use in youth: findings from the National Survey of Adolescents. J Trauma Stress. 2000;13(3):381-396.

33. Huang R, Ho SY, Wang MP, Lo WS, Lam TH. Sociodemographic risk factors of alcohol drinking in Hong Kong adolescents. $J$ Epidemiol Community Health. 2016;70(4):374-379.

34. Olweus D. The Revised Olweus Bully/Victim Questionnaire. Bergen, Norway: University of Bergen; 1996. 
35. Wu J, He Y, Lu C, et al. Bullying behaviors among Chinese schoolaged youth: a prevalence and correlates study in Guangdong Province. Psychiatry Res. 2015;225(3):716-722.

36. Birleson P. The validity of depressive disorder in childhood and the development of a self-rating scale: a research report. J Child Psychol Psychiatry. 1981;22(1):73-88.

37. Su L, Wang K, Zhu Y, et al. Norm of the depression self-rating scale for children in Chinese urban children. Chin Mental Health J. 2003;17(8):547-549.

38. Garver MS, Mentzer JT. Logistics research methods: employing structural equation modeling to test for construct validity. J Bus Logist. 1999;20:33-57.

39. Preacher KJ, Hayes AF. Asymptotic and resampling strategies for assessing and comparing indirect effects in multiple mediator models. Behav Res Methods. 2008;40(3):879-891.

40. Chen JH, Shiu CS. Sexual orientation and sleep in the U.S.: a national profile. Am J Prev Med. 2017;52(4):433-442.

41. Bernert RA, Hom MA, Iwata NG, Joiner TE. Objectively assessed sleep variability as an acute warning sign of suicidal ideation in a longitudinal evaluation of young adults at high suicide risk. J Clin Psychiatry. 2017;78(6):e678-e687.
42. Bernert RA, Joiner TE. Sleep disturbances and suicide risk: a review of the literature. Neuropsychiatr Dis Treat. 2007;3(6):735-743.

43. Pigeon WR, Pinquart M, Conner K. Meta-analysis of sleep disturbance and suicidal thoughts and behaviors. J Clin Psychiatry. 2012;73(9):e1160-e1167.

44. Hatzenbuehler ML. How does sexual minority stigma "get under the skin"? A psychological mediation framework. Psychol Bull. 2009;135(5):707-730.

45. Cacioppo JT, Hawkley LC, Berntson GG, et al. Do lonely days invade the nights? Potential social modulation of sleep efficiency. Psychol Sci. 2002;13(4):384-387.

46. Black WW, Fedewa AL, Gonzalez KA. Effects of "Safe School" programs and policies on the social climate for sexual-minority youth: a review of the literature. J LGBT Youth. 2012;9(4):321-339.

47. Kirmil-Gray K, Eagleston JR, Gibson E, Thoresen CE. Sleep disturbance in adolescents: sleep quality, sleep habits, beliefs about sleep, and daytime functioning. J Youth Adolesc. 1984;13(5):375-384.

48. Germain A, Richardson R, Moul DE, et al. Placebo-controlled comparison of prazosin and cognitive-behavioral treatments for sleep disturbances in US Military Veterans. J Psychosom Res. 2012;72(2):89-96.
Psychology Research and Behavior Management

\section{Publish your work in this journal}

Psychology Research and Behavior Management is an international, peerreviewed, open access journal focusing on the science of psychology and its application in behavior management to develop improved outcomes in the clinical, educational, sports and business arenas. Specific topics covered in the journal include: Neuroscience, memory and decision making; Behavior

\section{Dovepress}

modification and management; Clinical applications; Business and sports performance management; Social and developmental studies; Animal studies. The manuscript management system is completely online and includes a very quick and fair peer-review system, which is all easy to use. Visit http://www. dovepress.com/testimonials.php to read real quotes from published authors. 